\title{
BMJ Open Four reasons why too many informed consents to clinical research are invalid: a critical analysis of current practices
}

\author{
Anne Wisgalla (D) , Joerg Hasford ${ }^{1,2}$
}

To cite: Wisgalla A, Hasford J. Four reasons why too many informed consents to clinical research are invalid: a critical analysis of current practices. BMJ Open 2022;12:e050543. doi:10.1136/ bmjopen-2021-050543

- Prepublication history for this paper is available online. To view these files, please visit the journal online (http://dx.doi. org/10.1136/bmjopen-2021050543).

Received 23 February 2021 Accepted 09 November 2021
Check for updates

(c) Author(s) (or their employer(s)) 2022. Re-use permitted under CC BY-NC. No commercial re-use. See rights and permissions. Published by BMJ.

${ }^{1}$ Association of Medical Ethics Committees in Germany, Berlin, Germany

${ }^{2}$ Institute for Medical Information Processing, Biometry, and Epidemiology, Ludwig

Maximilians University Munich, Munich, Germany

Correspondence to Anne Wisgalla; geschaeftsstelle@akek.de

\section{ABSTRACT}

Objective Informed consent (IC) is a central ethical and legal requirement for clinical research that aims to protect the autonomy of participants. To enable an autonomous decision and valid consent, adequate understanding must be ensured. However, a considerable proportion of participants do not understand the relevant aspects about participation in research, for example, approximately $45 \%$ could not name at least one risk. As such, the inadequate understanding of IC has been known for several decades, and it still constitutes a severe problem for the ethical conduct of research. Through delineating the most pressing deficits of current IC procedures that lead to insufficient understanding, we aim to encourage the discussion among stakeholders, for example, clinical researchers, and to provide the grounds for practical solutions.

Main arguments (1) IC documents are too long to be read completely, thus, make it very difficult for potential participants to identify the material facts about the trial. (2) The low readability of the IC documents disadvantages persons with limited literacy. (3) The therapeutic misconception frequently prevents participants to realise that the primary purpose of clinical research is to benefit future patients. (4) Excessive risk disclosures, insufficient information about expected benefits and framing effects compromise a rational risk/benefit assessment.

Conclusion Due to these deficits, practices of IC in clinical research too often preclude adequate understanding of prospective participants, thus, invalidating IC. The gap between the well-specified ethical norm to enable IC and its insufficient translation into practice can no longer be accepted, as participant rights and the public trust in responsible research are at stake. Hence, immediate action is needed to address the prevailing deficits.

The ethical relevance of informed consent (IC) in healthcare and research is rooted in the appreciation for a person's autonomy, that is, the right and capability to develop own preferences and goals as well as to choose and act accordingly. As such, IC became a central ethical and legal requirement for medical research involving humans. In Germany, the first guidelines articulating the principle of consent in research were issued as early as 1900 and $1931 .{ }^{1}$ Yet, these guidelines did not prevent extensive crimes during medical experiments in Nazi Germany. In consequence, the verdict of the Nuremberg Doctors Trial emphasised as a first principle that: 'The voluntary consent of the human subject is absolutely essential'. ${ }^{2}$ The Nuremberg Code provided substantive groundwork for the concept of IC to evolve as an indispensable prerequisite for medical research. Today, the requirement of IC can be found in all international guidelines as well as in national and EU law. The Declaration of Helsinki in its current version defines the necessary elements of valid IC:' 25. Participation by individuals capable of giving informed consent as subjects in medical research must be voluntary. [...] 26. In medical research involving human subjects capable of giving informed consent, each potential subject must be adequately informed of the aims, methods, sources of funding, any possible conflicts of interest, institutional affiliations of the researcher, the anticipated benefits and potential risks of the study and the discomfort it may entail, post-study provisions and any other relevant aspects of the study. The potential subject must be informed of the right to refuse to participate in the study or to withdraw consent to participate at any time without reprisal. [...] After ensuring that the potential subject has understood the information, the physician or another appropriately qualified individual must then seek the potential subject's freely-given informed consent $[\ldots]$ '. ${ }^{3}$ Only if these elements are met can IC be regarded as valid.

Translation of these ethical requirements into practice is vital to safeguard participants' autonomy in clinical research. Systematic reviews, however, indicate that a considerable proportion of participants do not understand essential components of IC such as potential risks and benefits of the trial, procedures being applied (eg, randomisation), voluntariness of participation as well as nature and purpose of clinical research, that is, acquiring 
generalisable knowledge to benefit future patients. ${ }^{4-6}$ In this light, it must be assumed that current practices of IC considerably fail to deliver on the ethical standard of autonomy. Moreover, from a legal standpoint, the EU Clinical Trial Regulation that entered into force at the end of January 2022 sets an urgent need for improved IC practices to support participants' understanding. It states in Article 29(5) that 'it shall be verified that the subject has understood the information'. ${ }^{7}$ In the following we aim to dissect the most pressing insufficiencies of IC that cause inadequate understanding. Hereby, we hope to inspire the development of practical solutions in support for more self-determination of research participants.

\section{IC DOCUMENTS ARE TOO LONG}

In the last three decades, the average length of IC documents has increased 10 times with consequences on participants' understanding. ${ }^{8}$ A study that analysed IC documents of neuro-oncological randomized controlled trials (RCT) in 2011/2012 found an average word count of 7069 words and an average of 19 pages. ${ }^{9}$ Since then, the length of IC documents has continuously increased. In our work experience from research ethics committees, IC documents for oncological studies comprised of 40 pages plus additional five pages on data protection are common. Beardsley $e t \mathrm{al}^{10}$ already showed in 2007 that longer documents are associated with a lower level of understanding. According to this analysis, objective understanding, measured by the Quality of Informed Consent questionnaire, was increased when page count remained below seven pages. Sharp et $a l^{11}$ even concluded that IC documents should not exceed 1250 words, since longer documents are usually read incompletely. Similarly, the guidelines of the Council for International Organizations of Medical Sciences (CIOMS) of 2016 demand: 'The wording of the leaflet must be short and preferably not exceed two or three pages'. ${ }^{12}$

\section{LOW READABILITY OF IC DOCUMENTS HINDERS UNDERSTANDING OF PARTICIPANTS WITH LIMITED LITERACY}

Poor readability of IC documents is another reason attributing to limited understanding. According to the Survey of Adult Skills run by the Organisation for Economic Co-operation and Development (OECD) ${ }^{13}$ just half of the population of OECD member states $(51.2 \%)$ showed a higher proficiency in literacy allowing to understand multipage texts with high information density, whereas $15.5 \%$ had such limited reading skills that they were only able to capture isolated information in relatively short texts. The readability of IC documents is usually low as several studies using the Flesch-Kincaid scale indicate, thus, a high grade level is required to understand the assessed documents. ${ }^{814}$ Reinert et at ${ }^{\theta}$ classified eight of the nine IC documents examined as difficult or fairly difficult to read (eg, Flesh Reading Ease below 30); hence, only high school graduates or even university graduates will be able to understand the given information. Consequently, the complex language typically used in IC documents may prevent up to half of the population in OECD member states to access relevant information on their trial participation.

\section{THE THERAPEUTIC MISCONCEPTION IS WIDESPREAD}

In 1982, the therapeutic misconception was first addressed as a serious obstacle for proper understanding and valid IC in research with humans. ${ }^{15}$ While standard medical care focuses solely on the benefit for the individual patient, the primary goal of clinical research is to obtain generalisable knowledge on a specific scientific question, for example, which treatment is superior for a given disease. Thus, clinical trials are primarily oriented towards the benefit for future patients. A therapeutic misconception exists when a research participant fails to recognise the difference between regular healthcare and the participation in a clinical trial, therefore, assuming that treatment decisions are tailored only to their personal needs and/or holding disproportionate beliefs on their own personal benefit from participating in the trial, which is uncertain by nature. ${ }^{16}$ In the meta-analysis by Tam et $a l,{ }^{4} 37.6 \%$ of the participants showed a therapeutic misconception. Joffe et al $^{17}$ depicted that $30 \%$ of the participants in oncological studies assumed that the experimental treatment had already been proven as the most effective option. In the same study, only $46 \%$ of physicians recognised that the main goal of clinical trials is to gain scientific knowledge and benefit future patients. The therapeutic misconception presumably precludes participants' valid consent, since they lack correct views on the specific conditions of participation in research, which has direct implications for a proper assessment of benefits and risks. ${ }^{1819}$

In addition, participants often do not know that there are therapeutic alternatives outside the study. According to Tam et $a l^{4}$ only $64.1 \%$ of the participants understood that other therapies would be available if they withdrew from the study. In this context, a lack of understanding can have negative ramifications on the voluntariness of the participation, especially when participants fear that their withdrawal would have negative consequences such as insufficient treatment. Finally, misunderstandings may also give rise to fears of being treated inappropriately, either because participants hold the misconception that the clinical trial offers the best treatment and a discontinuation would cause insufficient treatment or because they think that physicians would treat them improperly or with less care if they withdrew or refused.

\section{POTENTIAL BENEFITS OF THE TRIAL PARTICIPATION ARE NOT SUFFICIENTLY ADDRESSED}

Another frequent shortcoming of IC documents in clinical research lies in an imbalanced description of possible risks versus expected benefits that often impedes a rational risk/benefit assessment. Essential but also extremely rare 
adverse effects are generally depicted in exceeding detail, whereas information on expected benefits remains vague and brief, often restricted to phrases like 'you may or may not benefit'. ${ }^{20}$ In our experience-that is, regularly assessing IC documents for research ethics approvalinformation on adverse effects can cover up to 12 pages out of a total of 40 pages, for example, for oncological trials that test new treatments. However, explanations on expected benefits usually do not exceed more than 12 lines. Kirby $e t a l^{21}$ reported that only one-third of the assessed IC documents mentioned specific potential benefits such as an expected delay of cancer progression; in these cases, information on benefits were usually found after description of adverse effects and presented by relatively shorter text. Along similar lines, the aforementioned study within a neuro-oncological research centre found that none of the analysed IC documents allowed a profound risk-benefit assessment. ${ }^{9}$ The emphasis on risks and legal aspects may seem comprehensible with regard to liability issues, but it does not justify the lack of adequate information on the expected benefit. A valid IC can only be given if a risk/benefit assessment is made possible through comprehensible and balanced information.

So far, little guidance exists on how to communicate potential benefits in the context of research where no benefits have reliably been established. Obviously, to avoid therapeutic misconception, information needs to emphasise the inherent uncertainty about personal benefits as well as refrain from overly optimistic wording. At the same time, vague notions of potential benefits do not suffice in weighing risks and benefits. Thus, experts demand that descriptions of potential benefits should at least specify the expected beneficial outcomes, for example, improvements of symptoms or survival. ${ }^{20}$ Moreover, Kahrass $e t a l^{20}$ also discuss different approaches to describe the likelihood of potential benefits. However, further investigation is necessary to determine best practices.

Additionally, almost no attention is paid to framing effects in risk disclosures for IC. A patient's or research participant's decision between two alternative options can substantially differ depending on whether a positive frame (eg, gains like survival rate related to a therapy) or a negative frame (eg, losses like mortality rate) is used to communicate risks. ${ }^{22}$ Consequently, framing effects can distort adequate understanding. Moreover, framing the likelihood of adverse effects negatively (eg, 10\% will experience fatigue vs positively framed, eg, $90 \%$ will not experience fatigue) can even affect participant wellbeing as it may contribute to increased fears and negative expectations that itself cause adverse effects in the sense of a nocebo effect. ${ }^{21} 23$

\section{DISCUSSION}

Given the four prevailing deficits in the practice of IC summarised here, it must be questioned whether research participants will find the pertinent information in the overlong, hardly readable IC documents and thus will be able to balance potential risks and expected benefits in agreement with their individual goals. Even if participants adequately capture the risks and benefits, they may not recognise how research substantially differs from standard clinical care, therefore, misconceiving the very nature of their participation. In conclusion, under the current circumstances, a significant proportion of participants do not understand the material facts about research, consequently precluding valid IC.

When speaking of validity, we refer to the ethical sense of IC that is grounded in its primary purpose to protect autonomy. ${ }^{24}$ In this sense, IC is valid if a person capable of deliberating own goals, free of controlling influences and with sufficient understanding intentionally decides about participating in a proposed trial, hence expresses an autonomous choice. However, a second sense of IC exists that aims at legally effective permissions of research procedures. ${ }^{2425}$ Laws and institutional practices in this sense are sometimes less rigorous regarding autonomy instead focussing on documented authorisation and detailed risk disclosure. Whereas consents under these conditions are legally valid and shield from liability, they often do not allow a participant's autonomous decision making.

At this point, a debate in research ethics that is relevant to our argument needs recognition. Ethicists have proposed dissenting views of what is required for valid IC, especially when it comes to the level of understanding a potential participant should have. On the 'minimalist account', necessary understanding is limited to a few items to make sure that a participant is consenting to a specific proposal, that is, comprehension of how to consent or refuse and of the procedures the person will undergo. ${ }^{26}$ The minimal understanding requirement appears to be tied to a rather narrow conception of respect for autonomy solely focusing on non-interference with a person's rights. We argue-following Beauchamp and Childress ${ }^{24}$ - that respect for autonomy poses more than a negative obligation to avoid rights violation and illegitimate control, it also contains a positive obligation to enable autonomous decisions through establishing meaningful understanding. We take both obligations as foundational and from that, derive what constitutes adequate understanding. It should not be misunderstood as a full understanding of every study detail. Rather, it is a grasp of core information that allow research participants to evaluate what they consent or refuse to and which advantages and disadvantages it might have, thus, allowing them to weigh the information against their interests and goals. What therefore needs to be understood for a valid IC is the nature and purpose of the research, its potential risks and benefits, applied procedures, right to refuse or withdraw and alternatives outside the study.

Despite efforts in support for better IC practices, the impact on participants' understanding has been relatively modest. Recent strategies to improve IC have focused for instance on documents with a short section on 'key information' as 
introduced by the revised US Common Rule, on multimedia approaches and on test/feedback interventions. However, effectiveness of some of these measures is still unclear, partly due to the high heterogeneity of studies on IC interventions, ${ }^{27} 28$ and strategies that show efficacy like enhanced IC documents ${ }^{27}$ are far from being implemented comprehensively. In result, the actual understanding of participants has not improved substantively over three decades. ${ }^{4}$ The challenges of IC clearly reach beyond the deficits outlined previously. ${ }^{25}{ }^{29}$ However, insufficient understanding remains a particularly pressing shortcoming as it dates back at least to the $1980 \mathrm{~s}^{30}$ and points to an unacceptable yet unresolved gap between the ethical principle of IC and its ineffective translation into practice. One acute force to act is set through the verification requirement in Article 29(5) of the EU Clinical Trial Regulation. ${ }^{7}$ Although the law became effective at the end of January 2022, some questions still need to be clarified, that is, by means of which methods shall understanding be verified and which parts of the IC document must be understood for a valid IC, as it is apparent that comprehension of a document of 10 pages or longer cannot be verified in detail.

A very fundamental obstacle affecting understanding lies in the increasing complexity of the issues to be disclosed for IC, such as complex study designs, novel methods like nextgeneration sequencing and processes like biobanking or data sharing. Usually, these concepts reach beyond the knowledge and experience of the general population and are thus intuitively difficult to understand and prone to misunderstandings.

The principle of respect for autonomy in medicine entails more than solely acknowledging the choices of patients and research participants. It also requires to actively encourage their decision making, for example, through providing pertinent information in an appropriate form or through resolving misconceptions when necessary. To achieve meaningful understanding, we urge for immediate action on IC practices. A few considerations may be helpful in doing so. First, IC should be regarded as a process rather than a form and, as such, will require a combination of interventions to improve understanding. According to a first meta-analysis, ${ }^{27}$ this needs to involve at least the development of understandable IC documents as well as strategies for substantive conversation between participants and the research team. Second, improving consent will also mean to engage patients and patient experts to a greater extent. Patient involvement facilitates IC processes that place informational needs and interests of participants in the centre, ${ }^{31}$ for example, in rendering IC documents more understandable and relevant through testing. ${ }^{32}$ To reach a broader implementation in practice, specific guidelines are necessary. Third, actions should also involve stakeholders who prepare written information, for example, sponsors and investigators, to shift emphasis on legal precautions to autonomy-driven consent and to identify barriers that hinder good practices. Fourth, robust evidence is required to select effective interventions for better understanding. Empirical research should thus adopt a cohesive definition of 'understanding' ${ }^{32}$ and standardised study designs. ${ }^{27}$ Finally, what is at stake if substantial change holds off are no less than the indispensable participant rights and ethical standards of legitimate medical research as well as the public trust in responsible research practices.

Contributors $\mathrm{JH}$ developed the concept of the article. AW reviewed the relevant literature and drafted the first version. AW and JH contributed substantially to the several versions of the manuscript. JH deceased before acceptance of the article. The final approval of the article was signed off by the surviving author AW.

Funding The authors have not declared a specific grant for this research from any funding agency in the public, commercial or not-for-profit sectors.

Competing interests None declared.

Patient consent for publication Not required.

Ethics approval This study does not involve human participants.

Provenance and peer review Not commissioned; externally peer reviewed.

Open access This is an open access article distributed in accordance with the Creative Commons Attribution Non Commercial (CC BY-NC 4.0) license, which permits others to distribute, remix, adapt, build upon this work non-commercially, and license their derivative works on different terms, provided the original work is properly cited, appropriate credit is given, any changes made indicated, and the use is non-commercial. See: http://creativecommons.org/licenses/by-nc/4.0/.

Author note AW is a research fellow at the Association of Medical Ethics Committees in Germany. She is a trained physician with a special interest in bioethics and scientific experience in the field of neuroimmunology. JH was professor for medical biometry and epidemiology with extensive experience with clinical trials. Since 1998, he served as a member of a Medical Ethics Committee and from 2012 to 2021 as president of the Association of Medical Ethics Committees in Germany (in an honorary function). As the informed consent (IC) materials and forms got longer and longer, and harder to read and understand, even for him, he realised that something has gone wrong with the current IC.

ORCID iD

Anne Wisgalla http://orcid.org/0000-0001-8900-0186

\section{REFERENCES}

1 Vollmann J, Winau R. Informed consent in human experimentation before the Nuremberg code. BMJ 1996;313:1445-7.

2 Shuster E. Fifty years later: the significance of the Nuremberg code. N Engl J Med 1997;337:1436-40.

3 World Medical Association. Declaration of Helsinki - Ethical Principles for Medical Research Involving Human Subjects. Fortaleza, Brazil, 2013. Available: https://www.wma.net/policiespost/wma-declaration-of-helsinki-ethical-principles-for-medicalresearch-involving-human-subjects/ [Accessed 9 Oct 2021].

4 Tam NT, Huy NT, Thoa LTB, et al. Participants' understanding of informed consent in clinical trials over three decades: systematic review and meta-analysis. Bull World Health Organ 2015;93:186-98.

5 Falagas ME, Korbila IP, Giannopoulou KP, et al. Informed consent: how much and what do patients understand? Am J Surg 2009;198:420-35.

6 Pietrzykowski T, Smilowska K. The reality of informed consent: empirical studies on patient comprehension-systematic review. Trials 2021;22:57.

7 European Parliament and Council of the European Union. Regualtion (EU) NO 536/2014 on clinical trials on medicinal products for human use, and Repealing directive 2001/20/EC. official Journal of the European Union, 2014.

8 Nathe JM, Krakow EF. The challenges of informed consent in HighStakes, randomized oncology trials: a systematic review. MDM Policy Pract 2019;4:238146831984032.

9 Reinert C, Kremmler L, Burock S, et al. Quantitative and qualitative analysis of study-related patient information sheets in randomised neuro-oncology phase III-trials. Eur J Cancer 2014;50:150-8.

10 Beardsley E, Jefford M, Mileshkin L. Longer consent forms for clinical trials compromise patient understanding: so why are they lengthening? J Clin Oncol 2007;25:e13-14.

11 Sharp SM. Consent documents for oncology trials: does anybody read these things? Am J Clin Oncol 2004;27:570-5.

12 Council for International Organizations of Medical Sciences. International ethical guidelines for health-related research involving humans. 4th Edition. Geneva, 2016. 
13 Organisation for Economic Co-operation and Development. OECD skills outlook 2013: first results from the survey of adult skills. OECD publishing, 2013.

14 Paasche-Orlow MK, Taylor HA, Brancati FL. Readability standards for informed-consent forms as compared with actual readability. $N$ Engl J Med 2003;348:721-6.

15 Appelbaum PS, Roth LH, Lidz C. The therapeutic misconception: informed consent in psychiatric research. Int J Law Psychiatry 1982;5:319-29.

16 Silverman $\mathrm{H}$. Protecting vulnerable research subjects in critical care trials: enhancing the informed consent process and recommendations for safeguards. Ann Intensive Care 2011;1:8.

17 Joffe S, Cook EF, Cleary PD, et al. Quality of informed consent in cancer clinical trials: a cross-sectional survey. Lancet 2001;358:1772-7.

18 Miller FG, Rosenstein DL. The therapeutic orientation to clinical trials. N Engl J Med 2003;348:1383-6.

19 Appelbaum PS, Lidz CW. Twenty-Five years of therapeutic misconception. Hastings Cent Rep 2008;38:5-6.

20 Kahrass H, Bossert S, Schürmann C, et al. Details of risk-benefit communication in informed consent documents for phase $\mathrm{I} / \mathrm{II}$ trials. Clin Trials 2021;18:71-80.

21 Kirby N, Shepherd V, Howick J, et al. Nocebo effects and participant information leaflets: evaluating information provided on adverse effects in UK clinical trials. Trials 2020;21:658.

22 Fridman I, Fagerlin A, Scherr KA, et al. Gain-loss framing and patients' decisions: a linguistic examination of information framing in physician-patient conversations. J Behav Med 2021;44:38-52.
23 Barnes K, Faasse K, Geers AL, et al. Can positive framing reduce nocebo side effects? current evidence and recommendation for future research. Front Pharmacol 2019;10:167.

24 Beauchamp TL, Childress JF. Principles of biomedical ethics. 8th edition. Oxford university press; 2019. Chapter 4, respect for autonomy, 2019: 99-154.

25 Grady C. Enduring and emerging challenges of informed consent. $N$ Engl J Med Overseas Ed 2015;372:855-62.

26 Millum J, Bromwich D. Informed consent: what must be disclosed and what must be understood? Am J Bioeth 2021;21:46-58.

27 Nishimura A, Carey J, Erwin PJ, et al. Improving understanding in the research informed consent process: a systematic review of 54 interventions tested in randomized control trials. BMC Med Ethics 2013;14:28

28 Soll D, Guraiib MM, Rollins NC, et al. Improving assent in health research: a rapid systematic review. BMC Med Res Methodol 2020;20:114.

29 Bolcato M, De Salvia A, Rodriguez D, et al. Is the Italian consent to transfusion really informed? A medico-legal analysis between old ghosts and new evidence. Transfus Apher Sci 2020;59:102823.

30 Cassileth BR, Zupkis RV, Sutton-Smith K, et al. Informed consent why are its goals imperfectly realized? N Engl J Med Overseas Ed 1980;302:896-900.

31 Health Research Authority / INVOLVE. Impact of public involvement on ethical aspects of research, 2016. Available: www.invo.org.uk/ posttypepublication/public-involvement-in-researchimpact-onethical-aspects-of-research [Accessed 9 Oct 2021].

32 Bossert S, Strech D. An integrated conceptual framework for evaluating and improving 'understanding' in informed consent. Trials 2017;18:482 